\title{
A UNIQUENESS THEOREM OF REFLECTABLE DEFORMATIONS OF A FUCHSIAN GROUP
}

\author{
JHARNA SENGUPTA
}

(Communicated by Irwin Kra)

\begin{abstract}
Let $\Gamma$ be a Fuchsian group of signature $\left(p, n, m ; \nu_{1}, \nu_{2}, \ldots, \nu_{n}\right)$; $2 p-2+m+\sum_{j=1}^{n}\left(1-1 / \nu_{j}\right)>0$. Let $I_{1}, I_{2}, \ldots, I_{m}$ be a maximal set of inequivalent components of $\Omega \cap \hat{\mathbf{R}} ; \Omega$ is the region of discontinuity and $\hat{\mathbf{R}}$ is the extended real line. Let $\phi$ be a quadratic differential for $\Gamma$. Let $f$ be a solution of the Schwarzian differential equation $S f=\phi$. If $\phi$ is reflectable, $f$ maps each $I_{j}$ into a circle $C_{j}$. For each $\gamma \in \Gamma$ there is a Moebius transformation $\chi(\gamma)$ such that $f \circ \gamma=\chi(\gamma) \circ f$. We prove that $\phi$ is determined by the homomorphism $\chi$ and the circles $C_{1}, C_{2}, \ldots, C_{m}$.
\end{abstract}

Introduction. Let $\Gamma$ be a Fuchsian group acting on the upper half plane $U$ and having signature $\left\{p, n, m ; \nu_{1}, \nu_{2}, \ldots, \nu_{n}\right\} ; n \geq 0, m \geq 0$ and $2 p-2+m+$ $\sum_{j=1}^{n}\left(1-1 / \nu_{j}\right)>0$. Let $\Omega$ be the region of discontinuity of $\Gamma$. Let $I_{1}, I_{2}, \ldots, I_{m}$ be a maximal set of inequivalent components of $\Omega \cap \hat{\mathbf{R}} ; \hat{\mathbf{R}}$ is the extended real line.

Let $\phi$ be a bounded reflectable quadratic differential for $\Gamma$ (will be defined below). Let $f$ be a meromorphic solution in $U$ of the Schwarzian differential equation

$$
S f=\phi .
$$

Then $f$ is a local homeomorphism of $U$ into the extended complex plane, and there exists a homomorphism $\chi$ of $\Gamma$ into the Moebius group such that

$$
f(\gamma(z))=\chi(\gamma)(f(z)), \quad \gamma \in \Gamma, z \in U .
$$

Moreover, $f$ maps each interval $I_{j}$ into a circle. We prove that $\phi$ is determined by the homomorphism $\chi$ and the circles on which $f\left(I_{j}\right)$ lie.

The theorem for the special case when $\Gamma$ is a covering group of a compact Riemann surface was first proven by Poincaré. A generalization of the theorem by Poincaré for a group representing a Riemann surface with punctures was proven by Kra [4]. The theorem for the case when $m=1$ and $f$ is real on $I_{1}$ was proven by Gallo and Porter [1]. They have also proved injectivity of an "extended monodromy map" (see the remark) for the case when $m>1$ in [2].

DEFINITIONS. The pair $(f, \chi)$ is a deformation of $\Gamma$ if (i) $f$ is a meromorphic local homeomorphism from $U$ onto an open subset of the extended complex plane and (ii) $\chi$ is a homomorphism of $\Gamma$ into the Moebius group such that

$$
f \circ \gamma=\chi(\gamma) \circ f \text { for all } \gamma \in \Gamma \text {. }
$$

Received by the editors June 25, 1987 and, in revised form, March 14, 1988.

1980 Mathematics Subject Classification (1985 Revision). Primary 30F 10, 30F20, 30F 30, 30F35, $35 \mathrm{~F} 40$.

(C) 1988 American Mathematical Society $0002-9939 / 88 \$ 1.00+\$ .25$ per page 
If $(f, \chi)$ is a deformation of $\Gamma$,

$$
\phi=S f=\left(\frac{f^{\prime \prime}}{f^{\prime}}\right)^{\prime}-\frac{1}{2}\left(\frac{f^{\prime \prime}}{f^{\prime}}\right)^{2}
$$

is a holomorphic quadratic differential for $\Gamma$; that is,

$$
\phi(\gamma(z)) \gamma^{\prime}(z)^{2}=\phi(z), \quad z \in U, \gamma \in \Gamma .
$$

It is a bounded quadratic differential, if

$$
\operatorname{Sup}\left\{(\operatorname{Im} z)^{2}|\phi(z)| ; z \in U\right\}<\infty .
$$

It is reflectable, if it has a holomorphic extension to $\Omega \cap \hat{\mathbf{R}}$ and it is real in $\Omega \cap \hat{\mathbf{R}}$.

A deformation $(f, \chi)$ is bounded reflectable if $S f$ is a bounded reflectable quadratic differential. It is easy to check that $f$ maps each $I_{j}$ into a circle if $S f$ is reflectable. We prove the following

THEOREM. Let $(f, \chi)$ and $(g, \chi)$ be two bounded reflectable deformations of a Fuchsian group $\Gamma$ of signature $\left\{p, n, m ; \nu_{1}, \nu_{2}, \ldots, \nu_{n}\right\} ; 2 p-2+m+\sum_{j=1}^{n}\left(1-1 / \nu_{j}\right)>$ 0 . If $f\left(I_{j}\right)$ and $g\left(I_{j}\right)$ lie on the same circle $C_{j}$ for each $j, j=1,2, \ldots, m$, then $f=g$.

ProOF. We take

$$
F(z)=\frac{(f(z)-g(z))^{2}}{f^{\prime}(z) g^{\prime}(z)}, \quad z \in U .
$$

It is easy to see that $F$ is holomorphic in $U$. Let $\chi(\gamma)=A_{\gamma}$ for $\gamma \in \Gamma$. We have, for $\gamma \in \Gamma$,

$$
\begin{aligned}
& f \circ \gamma=A_{\gamma} \circ f \\
& g \circ \gamma=A_{\gamma} \circ g .
\end{aligned}
$$

It is easy to check that for a Moebius transformation $B$,

$$
(B \circ f-B \circ g)^{2}=(f-g)^{2} B^{\prime}(f) B^{\prime}(g) .
$$

Using (1) and (2) one can easily prove that

$$
F(\gamma(z)) \gamma^{\prime}(z)^{-2}=F(z), \quad z \in U, \gamma \in \Gamma ;
$$

that is, $F$ is a $(-2)$ differential for $\Gamma$.

The functions $f$ and $g$ map each $I_{j} \subset \Omega \cap \hat{\mathbf{R}}$ into a circle $C_{j}$. For each $j$, there is a Moebius transformation $A_{j}$ mapping $C_{j}$ onto $\hat{\mathbf{R}}$. Using (2) we can show that

$$
\frac{\left(A_{j} \circ f-A_{j} \circ g\right)^{2}}{\left(A_{j} \circ f\right)^{\prime}\left(A_{j} \circ g\right)^{\prime}}=\frac{(f-g)^{2}}{f^{\prime} g^{\prime}}=F \text {. }
$$

Since $A_{j} \circ f$ and $A_{j} \circ g$ are real on each $I_{j}, F$ is real on each $I_{j}$. Since

$$
F(\gamma(z)) \gamma^{\prime}(z)^{-2}=F(z), \quad \gamma \in \Gamma,
$$

$F$ is real on $\Omega \cap \hat{\mathbf{R}}$. Therefore, $F$ extends to all of $\Omega$.

Next we study the behaviour of $F$ in a cusped region belonging to each puncture on $\Omega / \Gamma ; \Omega / \Gamma$ is a surface of type $(P, 2 n, 0), P=2 p+m-1$. We assume that $\gamma(z)=z+1$ determines a puncture. $\chi(\gamma)=A_{\gamma}$ is also parabolic since $S f$ is a 
bounded quadratic differential for $\Gamma[3]$. We replace $f$ and $g$ by $C \circ f$ and $C \circ g$, respectively, where $C$ is a fixed Moebius transformation. Then $A_{\gamma}$ is replaced by $C \circ A_{\gamma} \circ C^{-1}$ so that

$$
C \circ A_{\gamma} \circ C^{-1}(z)=z+b, \quad b \neq 0 .
$$

Following Kra [3] we have, for $z \in U$,

$$
f^{\prime}(z)=\sum_{n=0}^{\infty} a_{n} e^{2 \pi i n z}
$$

and

$$
g^{\prime}(z)=\sum_{n=0}^{\infty} b_{n} e^{2 \pi i n z} ; \quad a_{0}=b_{0}=b .
$$

Hence

$$
f(z)=b z+\sum_{n=1}^{\infty} A_{n} e^{2 \pi i n z}
$$

and

$$
g(z)=b(z)+\sum_{n=1}^{\infty} B_{n} e^{2 \pi i n z}
$$

for some $A_{n}$ and $B_{n}$. Therefore, we have

$$
F(z)=\frac{\left(\sum_{n=1}^{\infty} A_{n} e^{2 \pi i n z}-\sum_{n=1}^{\infty} B_{n} e^{2 \pi i n z}\right)^{2}}{\sum_{n=0}^{\infty} a_{n} e^{2 \pi i n z} \sum_{n=0}^{\infty} b_{n} e^{2 \pi i n z}}=\sum_{n=2}^{\infty} d_{n} e^{2 \pi i n z}
$$

for some $d_{n}$.

Following Kra [5], we define

$$
\operatorname{red} \operatorname{ord}_{Q} F=\frac{\operatorname{ord}_{Q} F}{\left|\Gamma_{Q}\right|}, \quad Q \in \Omega,
$$

where $\left|\Gamma_{Q}\right|$ is the order of the stabilizer $\Gamma_{Q}$ of $Q$, and for each parabolic fixed point $Q$ of $\Gamma$, we define

$$
\operatorname{red} \operatorname{ord}_{Q} F=r
$$

if the Fourier expansion of $F$ at $\infty$ is

$$
F(z)=\sum_{n=r}^{\infty} a_{n} e^{2 \pi i n z}, \quad a_{r} \neq 0, z \in \Omega .
$$

Let $\bar{D}_{0}$ be a fundamental set for $\Gamma$; that is, $\bar{D}_{0}$ is in one-to-one correspondence with the points in $\overline{\Omega / \Gamma}$ and $\bar{D}_{0} \subset \Omega \cup$ \{parabolic fixed points\}. Since $F$ is holomorphic in $\Omega$, red ord $F \geq 0$ in $\Omega$. By (3), $\operatorname{red~ord~}_{Q} F \geq 2$ for each parabolic fixed point $Q$. Hence, in general,

$$
\sum_{Q \in \bar{D}_{0}} \operatorname{red} \operatorname{ord}_{Q} F \geq 0
$$

notice that equality is possible when there is no parabolic fixed point. 
$F$ projects to a $(-2)$ differential $\hat{F}$ on $\Omega / \Gamma$. A direct calculation (as it is done in [5]) yields

$$
\begin{aligned}
& \sum_{Q \in \bar{D}_{0}} \operatorname{red} \operatorname{ord}_{Q} F \\
& =\sum_{X \in \overline{\Omega / \Gamma}} \operatorname{ord}_{X} \hat{F}-2\left(1-\frac{1}{\nu(X)}\right) ; \nu(X) \text { is the ramification order of } X \in \Omega / \Gamma \\
& =-2\left[(2 P-2)+\sum_{j=1}^{2 n}\left(1-\frac{1}{\nu_{j}}\right)\right] \\
& =-4\left[P-1+\sum_{j=1}^{n}\left(1-\frac{1}{\nu_{j}}\right)\right] \\
& =-4\left[2 p-2+m+\sum_{j=1}^{n}\left(1-\frac{1}{\nu_{j}}\right)\right]<0,
\end{aligned}
$$

since $2 p-2+m+\sum_{j=1}^{n}\left(1-1 / \nu_{j}\right)>0$ by our assumption. This proves that $F=0$; that is, $f=g$.

REMARK. In [2] Gallo and Porter have defined an extended monodrony mapping

$\Phi:\{$ Bounded reflectable differentials $\} \rightarrow$ Hom $\times K^{m}$,

where $K$ is the collection of circles in the extended complex plane $\hat{\mathbf{C}}$. For $(f, \chi)$ with $S f=\phi$, a bounded reflectable differential for $\Gamma, \Phi(\phi)=\left(\chi, C_{1}, C_{2}, \ldots, C_{m}\right)$ where $C_{j}$ is the circle in which $f\left(I_{j}\right)$ lies.

They have proved that the quotient of the image space under inner automorphisms of Aut $\hat{\mathbf{C}}$ is a real analytic manifold and the corresponding mapping is injective. The injectivity of this mapping is the same as our result in the following sense.

Let $\left(f, \chi_{1}\right)$ and $\left(g, \chi_{2}\right)$ be two bounded reflectable deformations of $\Gamma$. Assume that $f\left(I_{j}\right)=C_{j}$ and $g\left(I_{j}\right)=C_{j}^{\prime}$. If $\chi_{2}=A \chi_{1} A^{-1}$ and $C_{j}^{\prime}=A \circ C_{j}$ for some $A \in$ Aut $\hat{\mathbf{C}}$, then $g=A \circ f$; this follows from our results by taking

$$
F=\frac{(g-A \circ f)^{2}}{g^{\prime}(A \circ f)^{\prime}}
$$

ACKNowledgement. I want to thank Professor Irwin Kra of the State University of New York at Stony Brook for his assistance in preparing this work. I am grateful to Dipendra C. Sengupta of Elizabeth City State University for many helpful discussions and for his continual support.

\section{REFERENCES}

1. D. M. Gallo and R. M. Porter, Projective structures on open Riemann surfaces, Lecture Notes in Math., vol. 971, Springer-Verlag, Berlin and New York, 1981, pp. 36-47.

2. __ Extended monodromy mapping for bordered Riemann surfaces (to appear). 
3. I. Kra, Deformation of Fuchsian groups. II, Duke Math. J. 38 (1971), 499-508.

4. __ A generalization of a Theorem of Poincaré, Proc. Amer. Math. Soc. 27 (1971), 299-302.

5. __ Automorphic forms and Kleinian groups, Benjamin, Reading, Mass., 1972.

Department of Mathematics and Computer Science, Elizabeth City State UNIVERSity, Elizabeth City, NORTh CAROLINA 27909 